\begin{tabular}{|l|l|l|l|}
\hline Eiszeitalter u. Gegenwart & $\mathbf{3 1}$ & $\begin{array}{c}79-82 \\
\text { 1 Abb., } 1 \text { Tab., 1 Taf. }\end{array}$ & Hannover 1981 \\
\hline
\end{tabular}

\title{
Zwei Geschiebe von Riebeckit-Porphyr aus dem Geschiebemergel des Samlandes (Ostpreußen)
}

\author{
Horst-Jürgen Herbert \& Alfred Eisenack *)
}

Sampling (1924, 1936), boulder clay, erratic block, petrographic analysis, porphyre, Riebeckite, QAP-Diagram. Baltic Plain (Samland).

Zwei Geschiebe des Samländer Geschiebemergels sollen hier eine kurze Beschreibung finden. Die beiden Proben wurden von Eisenack 1924 und 1936 gesammelt und Herbert zur Bearbeitung überlassen. Die Fundpunkte, beide am Fuße der Geschiebemergel-Steilküste des Samlandes, liegen etwa $1 \mathrm{~km}$ südlich von Groß-Dirschheim, rd. $1 \mathrm{~km}$ voneinander entfernt.

Bei den Proben handelt es sich um zwei Geschiebe identisch im Habitus, 1,014 bzw. $3-4 \mathrm{~kg}$ schwer und mit einer Dichte von $2,65 \mathrm{~g} / \mathrm{cm}^{3}$ (Bestimmung von EISENACK). Die mikroskopische Untersuchung ergab, daß es sich bei den Gesteinen um pseudosphärolithische Riebeckit-Porphyre handelt, welche mit hoher Wahrscheinlichkeit der Ganggefolgschaft der nordländischen Alkaliprovinz zuzuordnen sind.

Gesteine dieser Art sind schon seit längerer Zeit bekannt. Eine gute petrographische Beschreibung von nordischen Leitgeschieben stammt von Hesemann (1936: 101). HeseMANN beschreibt einen pseudosphärolithischen Riebeckitgranophyr aus Mittenwalde, Kirchhain (Brandenburg), der den vorliegenden Gesteinen im wesentlichen gleicht. In einer rötlich-grauen bis braunen feinkörnigen Zwischenmasse liegen zahlreiche bis erbsengroße, sonnenartige Pseudosphärolithe mit ausgezeichneter Radialstruktur. Es sind Kugeln oder Ellipsoide von brauner bis braun-schwarzer Farbe, die einzeln oder zu nieren- und streifenförmigen Gebilden verwachsen vorkommen. Pseudosphärolithe sind nach der Definition von Rosenbusch (1910: 317) radialstrahlige Gebilde, die im wesentlichen die Zusammensetzung der Grundmasse haben und aus einem Gemenge von Quarzstrahlen und Feldspatstrahlen bestehen. Doch hat Hesemann Abbildungen von Schliffen nicht veröffentlicht.

Mikroskopisch ist in den Geschieben des Samlandes der Mineralbestand Orthoklas, Albit, Plagioklas, An27-32, Quarz, Riebeckit, Ägirinaugit und Erz zu erkennen. Die Werte der Modalanalyse sowie weitere Kenngrößen, wie Farbzahl und QAP-Werte sind in Tabelle 1 getrennt für Gesamtgestein, Zwischenmasse und Pseudosphärolithe angeführt.

Die Pseudosphärolithe (Taf. 1, Fig. 1,2) treten sowohl einzeln als auch in verzahnten Haufwerken auf. Die nahe Berührung bedingt oft ein verzerrtes Wachstum. Sie bestehen hauptsächlich aus kryptographisch bis mikrographisch verwachsenen Quarz-Feldspat-Kristallen mit sehr ausgeprägter radialstrahliger Anordnung. Bei gekreuzten Polarisatoren ist ein mehr oder weniger deutliches Interferenzkreuz zu beobachten. Gegen die Peripherie der Pseudosphärolithe geht die kryptographische Struktur in eine mikrographische über (Taf. 1, Fig. 3).

*) Anschrift der Verfasser: Prof. Dr. A. E is e n a ck, Geologisch-Paläontologisches Institut der Universität, Sigwartstr. 10, 7400 Tübingen 1; Dr. H.-J. H e r b e r t, Mineralogisch-Petrographisches Institut der Technischen Universität Clausthal, Adolf-Römer-Straße 2 A, 3392 Clausthal-Zellerfeld. 


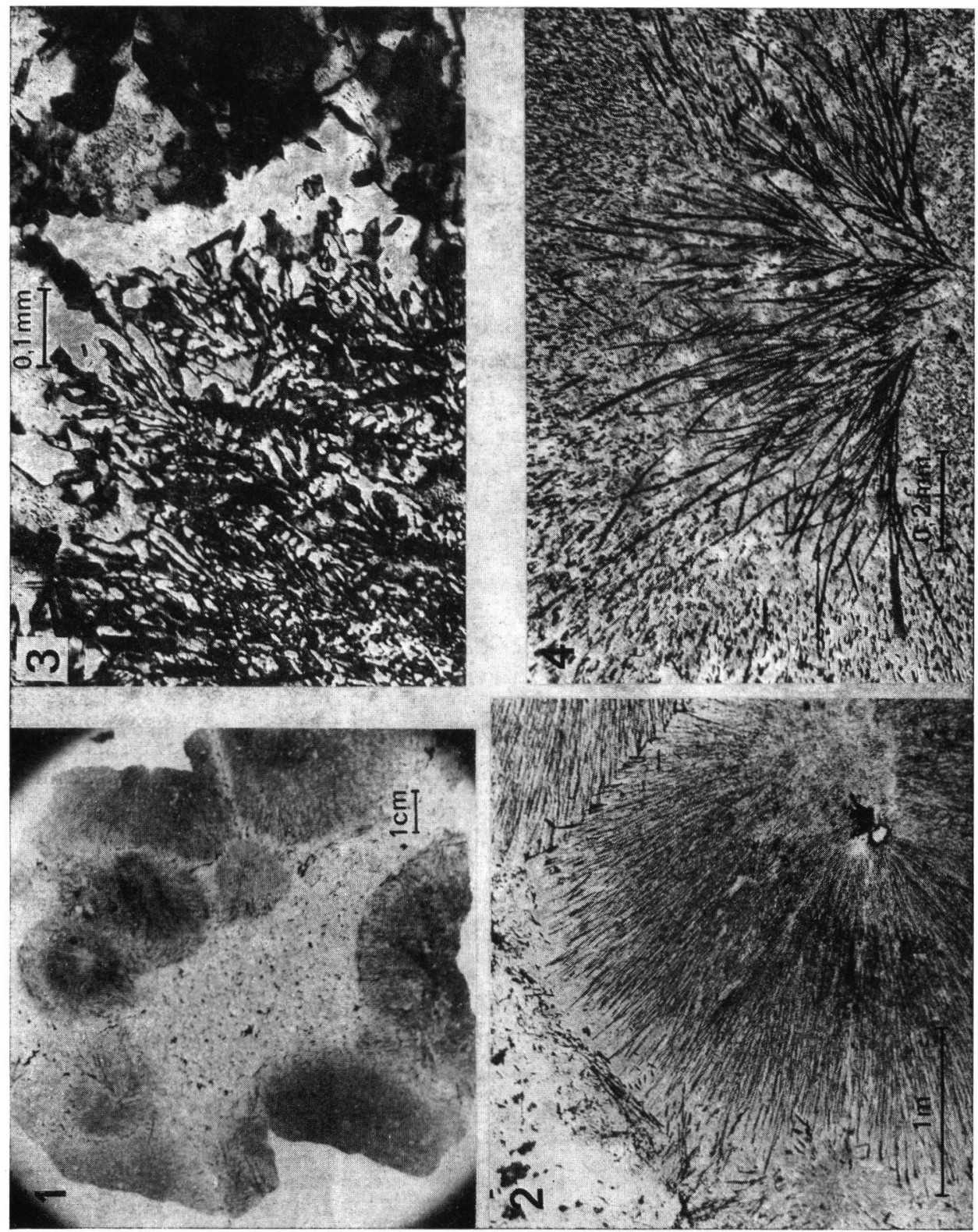

Tafel I

Fig. 1: Pseudosphärolithischer Riebeckit-Porphyr aus der Geschiebemergel-Steilküste des Samlandes, Ost-Preußen.

Fig. 2: Pseudosphärolith. In den Pseudosphärolithen sind die Riebeckit-Nadeln radialstrahlig angeordnet, im Randbereich oft tangential. (3,2 x 3,6 mm, // Pol.)

Fig. 3: Mikrographisch verwachsene Quarz-Feldspat-Aggregate mit radialstrahliger Anordnung im Randbereich der Sphärolithe. Der Übergang von der graphischen Struktur der Pseudosphärolithe zur granularen Struktur der Zwischenmasse tritt deutlich in Erscheinung. (0,9 x 0,6 mm, + Pol.).

Fig. 4: Büschelige, „eisblumenartige“ Riebeckit-Aggregate in den Pseudosphärolithen. $(1,4 \times 0,9 \mathrm{~mm}, / /$ Pol. $)$ 
Die markanteste Mineralphase der Kugelgebilde ist die Fe-reiche Riebeckit-Varietät Ossanit. Der Habitus der Riebeckitkristalle ist langsäulig bis nadelig, nach c gestreckt. Es treten sowohl regellos verstreute Einzelkristalle auf, als auch radialstrahlige Aggregate, oft mit büscheligem „eisblumenartigem" Wachstum (Taf. 1, Fig. 4). Während die Riebeckitnadeln in den Pseudosphärolithen radial angeordnet sind, liegen sie im Randbereich der Kugeln oft tangential (Taf. 1, Fig. 2). Die Spaltbarkeit (110) ist gut entwikkelt. Die Farbe ist in Schliffdicke intensiv mit starkem Pleochroismus; $\mathrm{X}=$ dunkel stahlblau, $\mathrm{Y}=$ braungelb, $\mathrm{Z}=$ dunkel blaugrau, fast opak. Die Lichtbrechung ist wegen des hohen Eisengehaltes sehr hoch. Die Auslöschungsschiefe $x \wedge c=0^{0}$. Der optische Achsenwinkel $2 \mathrm{~V}_{\mathrm{x}}=80^{\circ}-85^{\circ}$.

Als dunkle Gemengteile treten weiterhin vereinzelt Erzkörner auf, die den häufig exzentrisch gelagerten Kern der Pseudosphärolithe bilden.

Die Zwischenmasse zwischen den Pseudosphärolithen weist eine wesentlich gröberkörnige, und zwar eine granulare Struktur auf. Sie ist nicht mehr wie in den Pseudosphärolithen mikrographisch noch ist sie granophyrisch. Die Zwischenmasse besteht aus einem Mosaik aus Albit und Quarz. Ein basischerer Plagioklas An27-32 mit deutlicher Albitverzwillingung tritt nur untergeordnet auf. Vereinzelt sind große, idiomorphe, zersprungene, undulös auslöschende Hochquarze anzutreffen. Die dunklen Gemengteile werden von stark zersetztem Ägirinaugit, Riebeckit und Erz gebildet.

Tab. 1: Pseudosphärolithischer Riebeckit-Porphyr-Modalanalyse in Vol.- $\%$, Farbzahl und QAP-Werte

\begin{tabular}{|c|c|c|c|}
\hline & $\begin{array}{l}\text { Gesamt- } \\
\text { gestein }\end{array}$ & $\begin{array}{l}\text { Pseudo- } \\
\text { sphärolithe }\end{array}$ & $\begin{array}{l}\text { Zwischen- } \\
\text { masse }\end{array}$ \\
\hline Orthoklas & 30 & 30 & - \\
\hline Albit & 25 & 4 & 21 \\
\hline Plagioklas $\mathrm{An}_{27-32}$ & 3 & - & 3 \\
\hline Quarz & 21 & 3 & 18 \\
\hline Riebeckit (Ossanit) & 13 & 10 & 3 \\
\hline Ägirinaugit & 6 & - & 6 \\
\hline Erz & 2 & 1 & 1 \\
\hline Vol.- $0 \%$ & 100 & 48 & 52 \\
\hline$======$ & $=====$ & $========$ & $===ニ=ニ==-:$ \\
\hline Farbzahl & 21 & 22.9 & 19.2 \\
\hline Q & 26.6 & 8.2 & 42.8 \\
\hline A & 69.6 & 91.8 & 50.0 \\
\hline$P$ & 3.8 & - & 7.2 \\
\hline
\end{tabular}

Die untersuchten Geschiebe haben insgesamt eine granitische Zusammensetzung. Dies geht aus Tab. 1 und Abb. 1 deutlich hervor. Es sind jedoch auffällige Unterschiede in der Zusammensetzung von Pseudosphärolithen und Zwischenmasse festzustellen. Die Projektionspunkte der QAP-Werte der Zwischenmasse liegen im Granit-Feld, die der Pseudosphärolithe im Alkalisyenit-Feld (nach der Streckeisen-Nomenklatur für Tiefengesteine 1967: 160). 


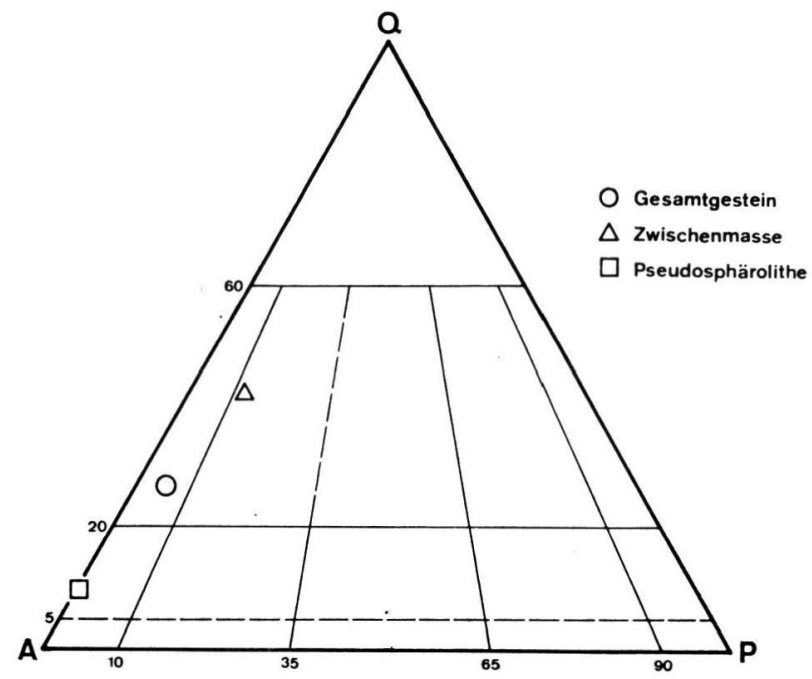

Abb. 1: Pseudosphärolithischer Riebeckit-Porphyr - QAP-Diagramm.

Die beschriebenen Gefügemerkmale und die Mineralparagenese lassen bei diesen Geschieben auf granitische Ganggesteine schließen. Geht man von der Nomenklatur von STRECKEISEN (1967: 175) aus (eine im deutschsprachigen Raum gebräuchliche Nomenklatur von Ganggesteinen, die ihrerseits auf JoHANNSEN 1917 zurückgeht), können die untersuchten Gesteine als Granitporphyre bzw. Riebeckit-Granitporphyre bezeichnet werden.

Riebeckit-Porphyr ist bisher 3 Mal gefunden worden, ein Mal von Hesemann (1936: 101) und $2 \mathrm{Mal}$ von Eisenack. Auffällig ist, daß die 2 letzten Fundpunkte nicht weit voneinander entfernt waren. Es handelt sich um ein seltenes aber sehr markantes Geschiebe, dessen Heimat vielleicht einmal aufgefunden wird. In diesem Falle würde es ein ausgezeichnetes Leitgeschiebe darstellen. Das rechtfertigt diesen kurzen Bericht.

Als Riebeckit-Porphyr wurde es bereits 1925 erkannt, und zwar durch Dr. A. PostelMANN (Königsberg), ein ausgezeichneter Kenner nordischer Geschiebe (mündl. Mitt.).

\section{Schriftenverzeichnis}

Johannsen, A. (1917): Suggestions for a quantitative mineralogical classification of igneous rocks. - J. Geol., 25: 63-97; Chicago.

Hesemann, J. (1936): Zur Petrographie einiger nordischer kristalliner Leitgeschiebe. - Abh.

Preuß. Geol. Landesanstalt, N. F., 173: 170-174; Berlin.

Rosenbusch, H. (1910): Elemente der Gesteinslehre. - 3. Aufl., 692 S.; Stuttgart (Schweizerbart). Streckeisen, A. L. (1967): Classification and Nomenclature of Igneous Rocks. - N. Jb. Miner. Abh., 107: 144-214; Stuttgart. 